\title{
Rheology as thermoanalytical method for polymers
}

\section{Special chapter containing 10 selected papers}

\author{
Markus Gahleitner
}

Received: 28 September 2009/ Accepted: 28 September 2009/Published online: 1 November 2009

(c) Akadémiai Kiadó, Budapest, Hungary 2009

Rheology, originating and developing in the area of polymer melts and solutions, has now broadened into many and often diverse segments of research. From food to cosmetics, from medical to biological applications, from mortars to adhesives-international conference events are frequent demonstrations of this diversification. In view of this broad scope, all papers in this volume the comparatively narrow area of polymer melts and solids. Nevertheless, with the present collection the authors demonstrate the versatility of rheological methods and concepts to understand and support various areas of polymer design and application development, from the basic molecular structure to end-use properties. The importance of understanding thermorheological and thermomechanical effects not only as a function of temperature, but also of thermal history and heating or cooling rate is maybe best expressed by the papers related to crystallization and processing effects.

I am especially glad that practically all papers have direct industrial relevance, and that several industrial scientists have contributed to this collection. This is also reflected by the need to combine or sometimes even integrate different metrological approaches for better and especially faster understanding the relevant aspects of material behavior demonstrated in many of the present papers. Let us hope that the positive examples presented here can serve as role models for multidisciplinary approaches to other imminent problems of polymer research.

Finally, let me thank the editors of JTAC for the invitation to assemble this special volume, the authors for their contributions and the reviewers for their helpful assistance and constructive critics. Scientific progress is team-play as much as it is competition, and we should never forget that!

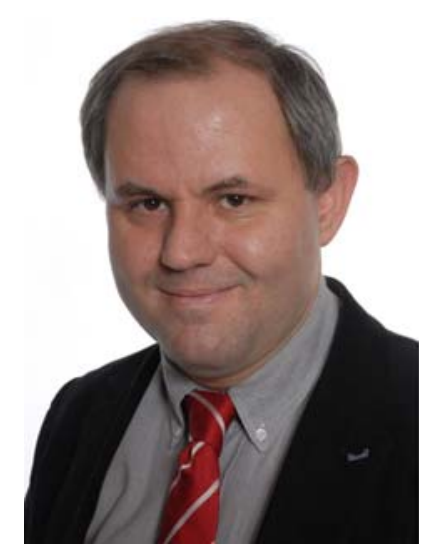

Dipl.-Ing.Dr.techn. Markus Gahleitner Guest Editor
M. Gahleitner $(\bowtie)$

Borealis Polyolefine GmbH, St. Peterstr. 25, 4021 Linz, Austria

e-mail: Markus.Gahleitner@borealisgroup.com 